Case

Report

\title{
A Case of Primary Mediastinal Leiomyosarcoma in Which Long-Term Survival Was Achieved
}

\author{
Yoshihito Iijima, MD, PhD, Hirohiko Akiyama, MD, PhD, Yuki Nakajima, MD, PhD, \\ Hiroyasu Kinoshita, MD, PhD, and Tomomi Hirata, MD, PhD
}

\begin{abstract}
Introduction: Primary leiomyosarcomas (LMS) of the mediastinum are extremely rare malignant mesenchymal tumors developing from soft tissues or great vessels. We present a case of a primary leiomyosarcoma of the middle mediastinum in which long-term survival was achieved.

Case Report: A 77-year-old man presented to us for examination with an extrapleural sign in his upper mediastinum on chest X-ray. Computed tomography (CT) revealed a well-circumscribed mass in the middle mediastinum. Thoracoscopic resection of the mediastinal tumor and immunohistological findings, which were positive for smooth muscle actin (SMA), HHF35 , vimentin, and desmin confirmed primary leiomyosarcoma. It recurred twice with solid right pulmonary metastases, which were resected. He was followed-up for a total of 9 years and 6 months from the first surgery with no signs of recurrence after his last surgery. Conclusion: Surgical resection of both the primary tumor and pulmonary metastases remains the mainstay of treatment of primary leiomyosarcomas.
\end{abstract}

Keywords: mediastinal tumor, leiomyosarcoma, pulmonary metastasis

\section{Introduction}

Primary leiomyosarcomas (LMS) of the mediastinum are extremely rare malignant mesenchymal tumors, which may develop from soft tissues of the mediastinum or great vessels. Mediastinal sarcomas including LMS account for $1.4 \%$ of soft tissue sarcomas ${ }^{1)}$ and about $3 \%-4 \%$ of mediastinal neoplasms. ${ }^{2,3)}$ Moreover, LMS accounts for $6.5 \%$ of malignant soft tissue tumors ${ }^{4)}$ with mediastinal LMS accounting for $0.15 \%$ of all

Division of Thoracic Surgery, Saitama Cancer Center, Saitama, Saitama Japan

Received: February 20, 2018; Accepted: February 27, 2018

Corresponding author: Yoshihito Iijima, MD, PhD. Division of Thoracic Surgery, Saitama Cancer Center, 780 Komuro, Ina-machi, Kita Adachi-gun, Saitama 362-0806, Japan

Email: yoshihito@cancer-c.pref.saitama.jp

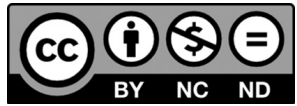

This work is licensed under a Creative Commons Attribution-NonCommercial-

NoDerivatives International License.

(C)2020 The Editorial Committee of Annals of Thoracic and Cardiovascular Surgery mediastinal tumors and $11 \%$ of primary mediastinal sarcomas. ${ }^{1)}$ In addition, mediastinal LMS have poor prognosis. To our knowledge, prior to this report, no cases of 5-year survival were reported in Japan. We present a rare case of LMS of the middle mediastinum in which long-term survival was achieved following three surgeries.

\section{Case Report}

A 77-year-old man who presented to our division for medical examination had an extrapleural sign in his upper mediastinum on chest X-ray. He had a history of hypertension, dyslipidemia, and prostate cancer. He had no respiratory symptoms and physical examination was unremarkable. Laboratory tests for tumor markers including carcinoembryonic antigen, squamous cell carcinoma antigen, and pro-gastrin-releasing peptide were within the normal ranges. Computed tomography (CT) of the chest (Fig. 1) and magnetic resonance imaging (MRI) revealed a well-circumscribed mass measuring $4.8 \times 2.9 \mathrm{~cm}$ with a relatively high contrast in the middle 


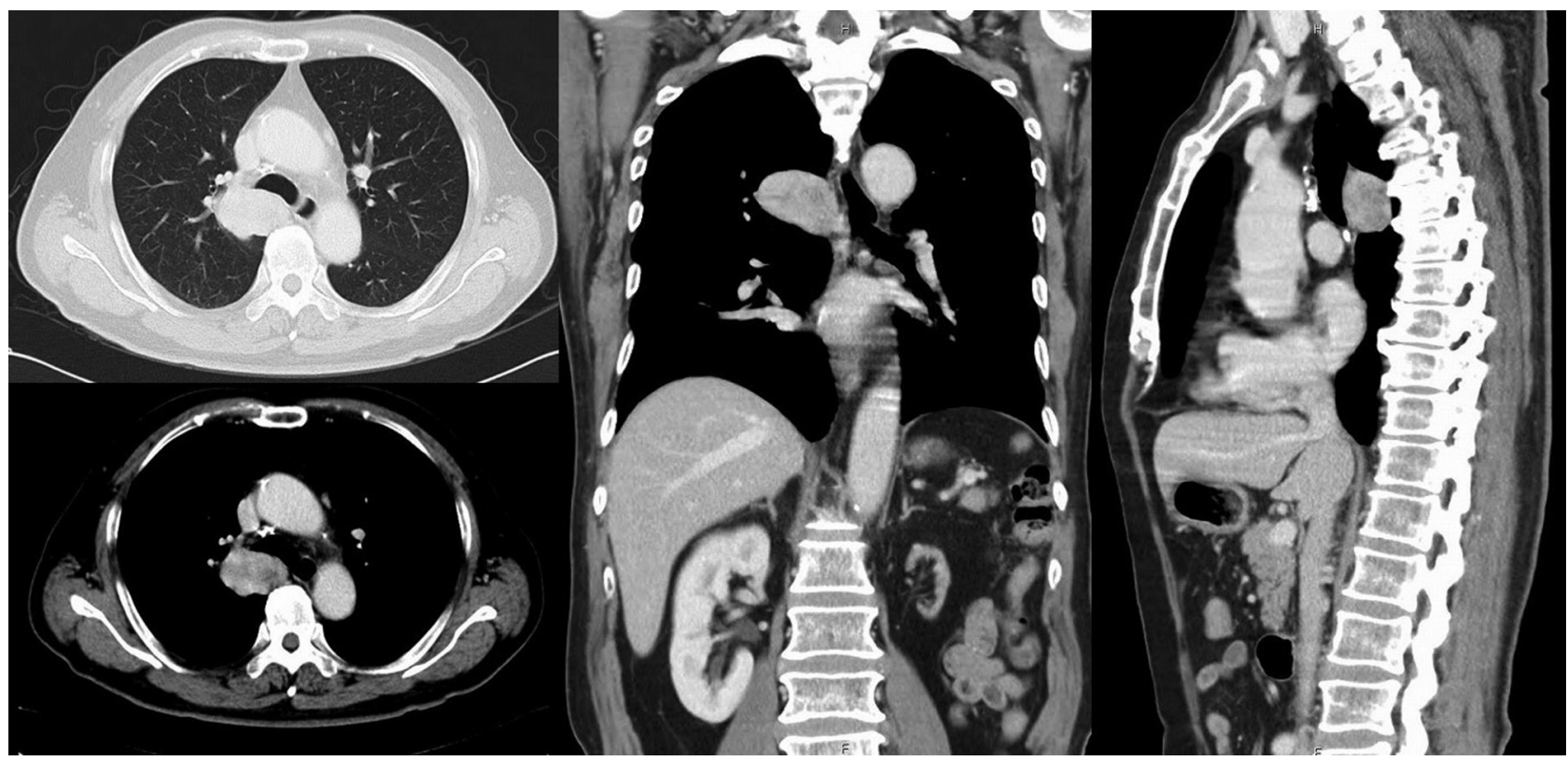

Fig. 1 Radiologic findings. CT scan showing a well-circumscribed mass measuring $48 \times 29 \mathrm{~mm}$ with a relatively high contrast in the middle mediastinum extending from the dorsal side of trachea to the right main bronchus and from the 5th to the 6th vertebral body. CT: computed tomography

mediastinum that extended from the dorsal side of trachea to the right main bronchus and from the 5th to the 6th vertebral bodies. A whole body scan using 2-deoxy-2-[18 $\mathrm{F}]$-fluorodeoxyglucose-positron emission tomography (FDG-PET)/CT demonstrated a maximum standardized uptake value of 3.17 during the early phase and 3.11 during the late phase only by the mediastinal tumor. A bronchoscopy revealed that the respiratory tract was compressed from the dorsal side by the tumor, which seemed to be a benign tumor; however, we were unable to reach a definitive diagnosis. A thoracoscopic resection of the mediastinal tumor was performed afterward for diagnosis and treatment. The tumor surface was smooth and it sparsely adhered to the lung. Since we were unable to detect the origin of the tumor and the continuous code from the ventral side of the tumor to the dorsal side of the superior vena cava was observed, a complete resection was performed (Fig. 2A). A frozen section analysis revealed a spindle cell tumor. However, it was difficult to determine whether it was benign or malignant. Macroscopically, the tumor was white, well-defined, and solid. Histopathological findings revealed moderate to high atypia in the irregular fascicles of the spindle cells (Fig. 2B), partial hemorrhage, and necrosis. Mitosis was 3 to 5/high power field (HPF). LMS was confirmed based on the immunohistological findings, which were positive for smooth muscle actin (SMA) (Fig. 2C), HHF-35, vimentin, and desmin and negative for CD34, S-100, CAM5.2, and AE1/AE3. Since the tumor involved destroying a relatively thick vein, it was suspected to originate from blood vessels (Fig. 2D). After the initial surgery, LMS recurred with solid pulmonary metastasis twice after 3 years and 8 months and again after 4 years and 6 months from the initial surgery. Partial pulmonary resection and basal segmentectomy of the right lung was performed each time. The patient was followed-up for a total of 9 years and 6 months from the first surgery with no signs of recurrence from the last surgery.

\section{Discussion}

LMS of the mediastinum is an extremely rare tumor with only 19 cases reported in Japan including our case (Table 1) ${ }^{5-12)}$ The patients' ages ranged from 34 to 84 years (mean: 63.4 years) of which 10 were females and 9 were males. Reports included three lesions in the anterior, four in the superior, seven in the middle, and five in the posterior mediastinum. The tumor sizes ranged from 4.0 to $21.0 \mathrm{~cm}$ (mean: $9.6 \mathrm{~cm}$ ). Surgery was the primary treatment in 13 patients. In total, six patients were deemed inoperable. The tumors originated from great vessels including the superior vena cava in 4 patients, while the origin was unknown in the remaining 15 patients. A total of nine patients were treated by surgical 


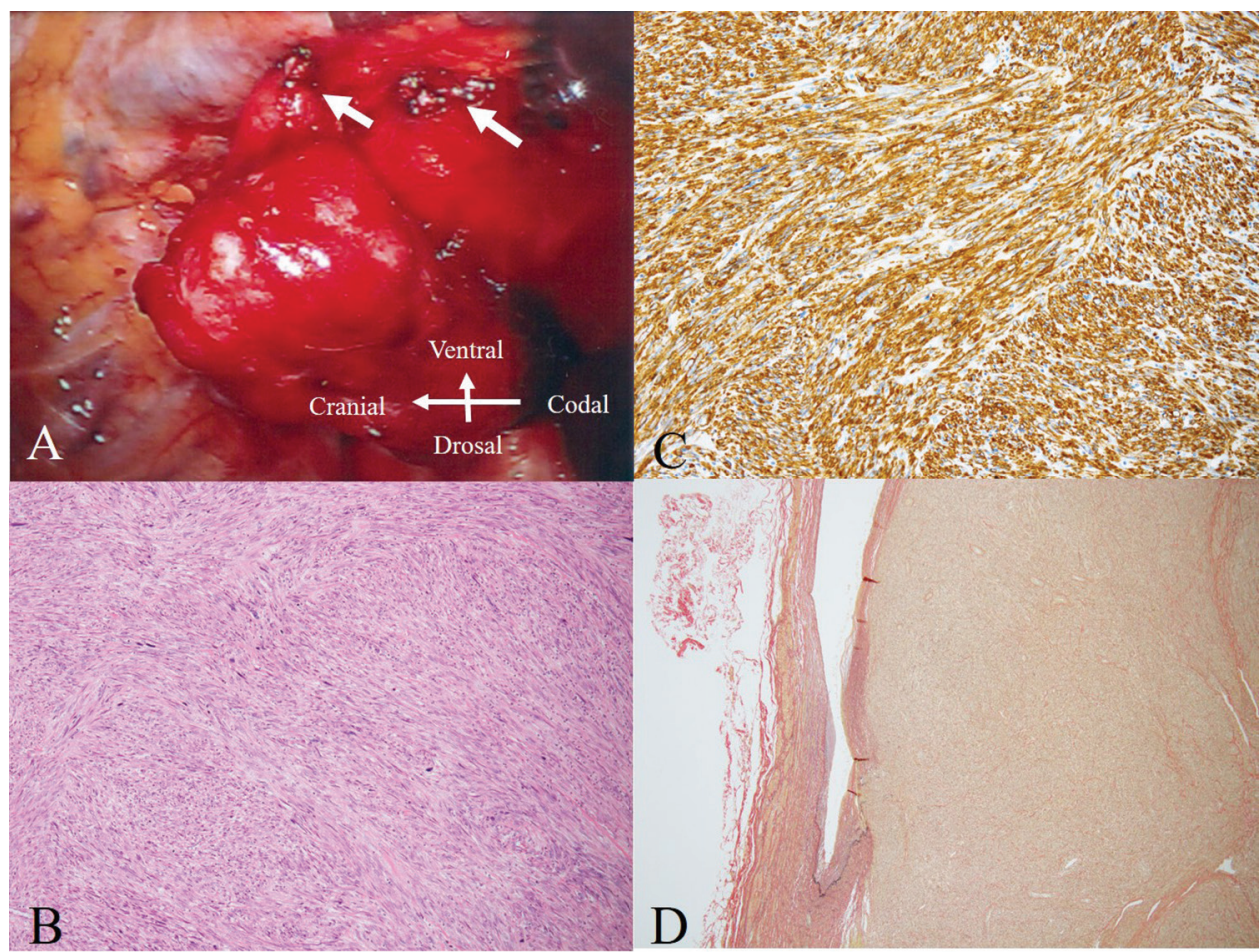

Fig. 2 Intra-operative findings and microscopic findings. (A) The tumor surface is smooth and the continuous code from the ventral side of the tumor to the dorsal side of the superior vena cava is observed (white arrows). (B) Histopathological findings show moderate to high atypia in the irregular fascicles of the spindle cells, partial hemorrhage, and necrosis. Hematoxylin and eosin staining. Magnification, $\times 10$. (C) Tumor cells are positive for SMA. Immunohistochemical staining for SMA. Magnification, $\times 20$. (D) The tumor involves destroying a relatively thick vein, suggesting that it originates from blood vessels. VerhoeffVan Gieson elastic staining. Magnification, $\times 4$. SMA: smooth muscle actin

resection alone with one patient receiving chemoradiation therapy in addition to surgery, two patients receiving radiotherapy either before or after surgery, and one receiving chemotherapy after surgery. Whereas four patients received chemotherapy and/or radiotherapy for their primary treatment and two patients opted for best supportive care following their diagnosis.

Although the prognosis of mediastinal LMS is unknown, an analysis by Burt and colleagues ${ }^{1)}$ of 47 cases of patients with primary mediastinal sarcomas including five with LMS revealed a median survival of 13 months with a 5 -year overall survival of $32 \%$. In that series, the five patients with LMS were classified as having high-grade tumors was based on cellular differentiation, the degree of tumor cellularity and vascularity, amount of necrosis, and the number of mitoses. The 5-year survival rate among those was $27 \%$. Complete resection was reported to be the most significant factor correlating with survival in patients with LMS.111-13) Additionally, a recent metaanalysis revealed the efficacy of adjuvant chemotherapy with doxorubicin and ifosfamide in those patients. ${ }^{14)}$ In our case, we performed complete resection of the primary lesion; however, the patient did not receive adjuvant chemotherapy.

The lung is the most frequent site of metastasis from soft tissue sarcomas with complete surgical resection being the most consistent predictor of long-term survival in these patients. ${ }^{15)}$ In Japan, out of 19 reported cases, 8 had metastases synchronous or metachronous to various organs with pulmonary metastases noted in 5 cases.

Chemotherapy should be considered in cases where complete resection cannot be performed. In metastatic cases, doxorubicin is the only drug with an established efficacy as a single agent. ${ }^{16)}$ Recently, newly developed drugs including pazopanib, ${ }^{17,18)}$ trabectedin, ${ }^{19)}$ and eribu$\operatorname{lin}^{20)}$ have attracted attention for the treatment of soft tissue sarcomas. Although there are no reports on their use for the treatment of mediastinal sarcomas, similar outcomes are expected. Complete resection is important for treatment of both the primary lesion of the mediastinal 
Table 1 Case reports of mediastinal leiomyosarcoma in Japan

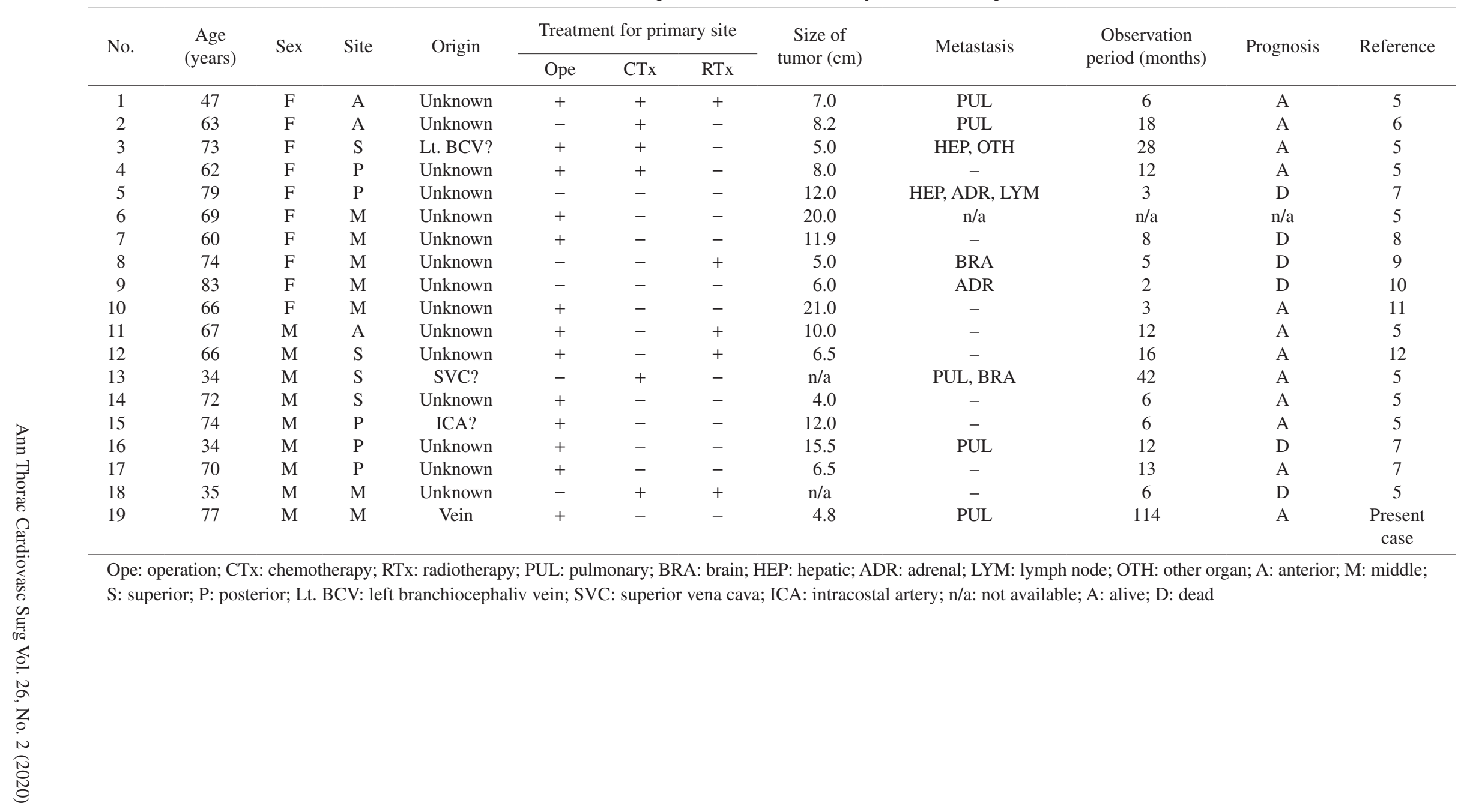


LMS and lung metastasis. A better prognosis is expected with complete resection. Our case had a long-term survival of 9 years and 6 months despite the recurrence of solid lung metastases twice following the initial resection of the primary lesion. This suggests that long-term survival can be achieved with complete resection despite the recurrence of pulmonary metastases.

\section{Conclusion}

Mediastinal LMS is an extremely rare neoplasm. Complete surgical resection of the primary tumor is a significant prognostic factor. Despite limited data on the management of mediastinal LMS, surgical resection of solid pulmonary metastases remains the mainstay of treatment.

\section{Consent}

A written informed consent was provided by the patient for publication of this report.

\section{Acknowledgment}

We would like to thank Dr. Yu Nishimura of Division of Pathology at Saitama Cancer Center for her valuable input.

\section{Disclosure Statement}

The authors report no conflicts of interest.

\section{References}

1) Burt M, Ihde JK, Hajdu SI, et al. Primary sarcomas of the mediastinum: results of therapy. J Thorac Cardiovasc Surg 1998; 115: 671-80.

2) Shields TW, Robinson PG. Mesenchymal tumors of the mediastinum. In: Shields TW, LoCiero J, Ponn RB, Rusch VW eds.; General thoracic surgery. 7th ed. Philadelphia: Lippincott Williams \& Wilkins, 2009; pp 2473-97.

3) Silverman NA, Sabiston DC. Mediastinal masses. Surg Clin North Am 1980; 60: 757-77.

4) Ogura K, Higashi T, Kawai A. Statistics of soft-tissue sarcoma in Japan: report from the bone and soft tissue tumor registry in Japan. J Orthop Sci 2017; 22: 755-64.

5) Abe M, Okashiwa H, Takeoka T. A case of mediastinal leiomyosarcoma with recurrent metastases. J Jpn Surg Assoc 2001; 62: 2644-7. (in Japanese)

6) Ono K, Takeda T, Fujinami M, et al. A case of anterior mediastinal leiomyosarcoma with multiple lung metastases and bone metastasis treated by chemotherapy. AJRS 2013; 2: 742-5. (in Japanese)
7) Abiko T, Sato S, Futamata T, et al. A case of pleomorphic leiomyosarcoma of the posterior mediastinum. J Jpn Assoc Chest Surg 2005; 19: 819-22. (in Japanese)

8) Hirano H, Kizaki T, Sashikata T, et al. Leiomyosarcoma arising from soft tissue tumor of the mediastinum. Med Electron Microsc 2003; 36: 52-8.

9) Shundo Y, Takahashi T, Itaya T, et al. [Leiomyosarcoma of the middle mediastinum; report of a case]. Kyobu Geka 2011; 64: 603-5. (in Japanese)

10) Yoshida M, Ando S, Naito Y, et al. Mediastinal leiomyosarcoma concurrent with intra-aortic thrombosis. BMJ Case Rep 2013; pii: bcr2012007527.

11) Chen F, Muranishi Y, Minakata K, et al. Complete resection of a giant mediastinal leiomyosarcoma. Ann Thorac Surg 2015; 99: e69-71.

12) Iwata $T$, Miura $T$, Inoue $K$, et al. Primary leiomyosarcoma of the anterior mediastinum encasing the aortic arch, left common carotid and left subclavian arteries. Ann Thorac Cardiovasc Surg 2012; 18: 140-3.

13) Moran CA, Suster S, Perino G, et al. Malignant smooth muscle tumors presenting as mediastinal soft tissue masses. A clinicopathologic study of 10 cases. Cancer 1994; 74: 2251-60.

14) Pervaiz N, Colterjohn N, Farrokhyar F, et al. A systematic meta-analysis of randomized controlled trials of adjuvant chemotherapy for localized resectable soft-tissue sarcoma. Cancer 2008; 113: 573-81.

15) Billingsley KG, Burt ME, Jara E, et al. Pulmonary metastases from soft tissue sarcoma: analysis of patterns of diseases and postmetastasis survival. Ann Surg 1999; 229: 602-10; discussion 610-2.

16) van der Graaf WT, Blay JY, Chawla SP, et al. Pazopanib for metastatic soft-tissue sarcoma (PALETTE): a randomised, double-blind, placebo-controlled phase 3 trial. Lancet 2012; 379: 1879-86.

17) Bramwell VH, Anderson D, Charette ML, et al. Doxorubicin-based chemotherapy for the palliative treatment of adult patients with locally advanced or metastatic soft tissue sarcoma. Cochrane Database Syst Rev 2003; CD003293.

18) Nakamura T, Matsumine A, Kawai A, et al. The clinical outcome of pazopanib treatment in Japanese patients with relapsed soft tissue sarcoma: a Japanese musculoskeletal oncology group (JMOG) study. Cancer 2016; 122: 1408-16.

19) Demetri GD, von Mehren M, Jones RL, et al. Efficacy and safety of trabectedin or dacarbazine for metastatic liposarcoma or leiomyosarcoma after failure of conventional chemotherapy: results of a phase iii randomized multicenter clinical trial. J Clin Oncol 2016; 34: 786-93.

20) Schöffski P, Chawla S, Maki RG, et al. Eribulin versus dacarbazine in previously treated patients with advanced liposarcoma or leiomyosarcoma: a randomised, open-label, multicentre, phase 3 trial. Lancet 2016; 387: 1629-37. 